\title{
Stratification based on methylation of TBX2 and $T B X 3$ into three molecular grades predicts progression in patients with pTa-bladder cancer
}

\author{
Willemien Beukers ${ }^{1}$, Raju Kandimalla ${ }^{1}$, Roy G Masius ${ }^{1}$, Marcel Vermeij ${ }^{1}$, Ries Kranse ${ }^{2}$, \\ Geert JLH van Leenders ${ }^{1}$ and Ellen C Zwarthoff ${ }^{1}$
}

${ }^{1}$ Department of Pathology, Erasmus MC, Rotterdam, The Netherlands and ${ }^{2}$ Dutch Cancer Registry, Rotterdam, The Netherlands

\begin{abstract}
The potential risk of recurrence and progression in patients with non-muscle-invasive bladder cancer necessitates followup by cystoscopy. The risk of progression to muscle-invasive bladder cancer is estimated based on the European Organisation of Research and Treatment of Cancer score, a combination of several clinicopathological variables. However, pathological assessment is not objective and reproducibility is insufficient. The use of molecular markers could contribute to the estimation of tumor aggressiveness. We recently demonstrated that methylation of GATA2, TBX2, TBX3, and ZIC4 genes could predict progression in Ta tumors. In this study, we aimed to validate the markers in a large patient set using DNA from formalin-fixed and paraffin-embedded tissue. PALGA: the Dutch Pathology Registry was used for patient selection. We included 192 patients with pTaG1/2 bladder cancer of whom 77 experienced progression. Methylation analysis was performed and log-rank analysis was used to calculate the predictive value of each methylation marker for developing progression over time. This analysis showed better progression-free survival in patients with low methylation rates compared with the patients with high methylation rates for all markers $(P<0.001)$ during a followup of ten-years. The combined predictive effect of the methylation markers was analyzed with the Coxregression method. In this analysis, TBX2, TBX3, and ZIC4 were independent predictors of progression. On the basis of methylation status of TBX2 and TBX3, patients were divided into three new molecular grade groups. Survival analysis showed that only $8 \%$ of patients in the low molecular grade group progressed within 5 years. This was 29 and $63 \%$ for the intermediate- and high-molecular grade groups. In conclusion, this new moleculargrade based on the combination of TBX2 and TBX3 methylation is an excellent marker for predicting progression to muscle-invasive bladder cancer in patients with primary pTaG1/2 bladder cancer.

Modern Pathology (2015) 28, 515-522; doi:10.1038/modpathol.2014.145; published online 14 November 2014
\end{abstract}

Bladder cancer is the fifth most common malignancy in the western world and presents a significant health problem. ${ }^{1}$ Clinically, $75-80 \%$ of bladder tumors are diagnosed as non-muscle-invasive disease (Ta, T1, and Tis), while the remaining part is muscle invasive (T2-T4). Ta tumors represent the largest group of non-muscle-invasive disease, accounting for $\sim 70 \% .^{2}$ These tumors are limited to the urothelial layer and are treated by transurethral resection. Overall, prognosis of these patients is

Correspondence: Dr EC Zwarthoff, PhD, Department of Pathology, Erasmus MC, PO Box 2040, 3000 CA Rotterdam, The Netherlands. E-mail: e.zwarthoff@erasmusmc.nl

Received 24 March 2014; revised 4 September 2014; accepted 6 September 2014; published online 14 November 2014 favorable, especially for patients with G1/G2 disease. Although, high recurrence rates are observed, progression rates in patients with primary $\mathrm{pTaG} 1 / 2$ bladder cancer remain limited, ranging from $1 \%$ to6\%. ${ }^{3,4}$ Yet, due to the high mortality rates associated with muscle-invasive bladder cancer, a life-long followup by cystoscopy is required. Cystoscopy is an expensive and invasive procedure, causing pain and discomfort to the patient. ${ }^{5}$ The long survival of non-muscle-invasive bladder cancer patients makes bladder cancer one of the most costliest cancers. ${ }^{6}$ Costs and patient burden could be reduced by lowering the number of cystoscopies in patients with pTaG1/2 disease. Hence, correct risk stratification is necessary in order to safely adjust the surveillance protocol. 
Currently, the risk of progression is highly based on the pathological review. Yet, the reproducibility of pathology is modest and this may result in unwanted variations in patient management. ${ }^{2,7-9}$ Therefore, many studies have been performed investigating new prognostic markers for the prediction of progression. ${ }^{10-12}$ Nevertheless, there are still no accurate biomarkers available to predict progression in this large subgroup of patients.

Epigenetic aberrations, such as DNA hypermethylation, have an important role in the formation of many carcinomas and may serve as a cancer biomarker in the prediction of disease progression. ${ }^{13-15}$ Recently, Kandimalla et al. ${ }^{16}$ studied genome-wide hypermethylation of $\mathrm{CpG}$ islands in association with tumor progression. They investigated 238 unique CpG islands in pTaG1/2 tumors with and without disease progression, ie progression to muscle-invasive disease, metastasis or dead of disease. Four different CpG islands were selected, that were significantly associated with tumor progression, ie GATA2, TBX2, TBX3, ZIC4. In this study, the aim was to validate the clinical relevance of these four selected markers in a large set of patients with pTaG1/2 bladder cancer with and without progression.

\section{Materials and methods}

\section{Patient and Tissue Collection}

Patients with primary pTaG1/G2 bladder cancer were included. The nationwide network and registry of histopathology and cytopathology in The Netherlands (PALGA) ${ }^{17}$ was searched for patients with progression of disease after resection of a primary pTaG1/G2 tumor and the tissue block and patient data were requested from the indicated pathology archives. Tumor tissue was used according to the code of secondary use of human tissue (www.federa.org). Progression was defined as progression to muscle-invasive disease, metastases or dead of disease. Patients with pTaG1/G2 disease without progression were retrieved from our own pathology archive. All available cases were reevaluated by an expert uropathologist (GvL).

\section{DNA Isolation and Methylation Analysis}

Formalin-fixed paraffin-embedded tumor tissue was manually dissected from $20 \mu \mathrm{m}$ tissue sections based on an accompanying HE-stained section. Tumor tissues were dewaxed with xylene and ethanol. DNA was extracted using the QIAamp mini and Blood kit (Qiagen) according to manufacturer's protocol.

Methylation analysis of GATA2, TBX2, TBX3, and ZIC4 was performed using the EZ DNA MethylationGold Kit (Zymo Research Corporation, Irvine, CA, USA) according to the manufacturer's protocol. As described by Kandimalla et al., ${ }^{16}$ DNA was treated with sodium bisulfite, followed by a bisulfitespecific PCR for the four regions of interest. For each PCR reaction a DNA input of $10 \mathrm{ng}$ and a PCR primer concentration of $20 \mathrm{pM}$ was required. After PCR, a SNaPshot analysis was performed, using probes that annealed to the PCR product adjacent to the cytosine of interest. Probes were extended with a labeled dideoxynucleotide and the products were analyzed on an automatic sequencer (ABI PRISM 3100 Genetic Analyzer, Applied Biosystems), with the label indicating the presence or absence of a methylated cytosine. Primers and probes are given in Table 1. For each gene, the methylation percentage was calculated by dividing the height of the methylated peak by the sum of the height of the methylated and unmethylated peaks multiplied by $100 \%{ }^{16}$

\section{Statistical Analysis}

Statistical analyses were completed using the IBM SPSS statistics 20. To determine relationships between different variables $\chi^{2}$ test was used. Survival was

Table 1 Primer and probe sequences

\section{BSP primers}

ZIC4

TBX2

TBX3

GATA2
TTTTTATTTTTTGAGGGTAAATATTTAGTAATCTCCAAAAAACCTCTAAAACAAC GTGGGGTTTTGGAATTTAGAATAGTAACCACAAACTTAACCATCCACTAC TTTGTTAATGGTTTGTAAATTTAAAATAAATTTATCACCCACC TATTGTTTTGTGTTTTTGGGAACAAATTACAAACAAATTATACCTAA

SNaPshot probes

\begin{tabular}{llcccc}
\hline Probe & Sequence $\left(5^{\prime} \rightarrow 3^{\prime}\right)$ & Size $(b p)$ & Strand & $U M$ & $M T$ \\
\hline ZIC4 & T10 TAAATTTTGTTTAAATTTTT & 30 & Sense & T & C \\
TBX2 & T45 GTTGATGGATATTGTAGT & 63 & Sense & T & C \\
TBX3 & T13 AATTTTGGGATTAAAA & 30 & Sense & T & C \\
GATA2 & T20 ACAAACAAATTATACCTAAC & 40 & Antisense & A & G \\
\hline
\end{tabular}

Abbreviation: BSP, bisulfite-specific PCR.

${ }^{\mathrm{a} C o n c e n t r a t i o n}$ in reaction. 
estimated according to the Kaplan-Meier method and compared using log-rank tests. Multivariate Cox-regression analysis with backward elimination was used to calculate the independent prognostic value of the methylation markers. $P$-values $<0.05$ were considered statistically significant.

\section{Results}

\section{Patients and Tumor Characteristics}

A total of 192 patients with primary pTaG1/G2 bladder cancer were included in this study. There were 115 patients without progression with a median followup of 84 months. 77 patients had progression of disease with a median time to progression of 38 months. Of these, 66/77 (86\%) patients developed progression within 10 years. Patient and tumor characteristics are depicted in Table 2. Male:female ratio in both groups was 3:1. Overall, primary tumors of the progressors were higher grade (G2) compared with the primary tumors of the non-progressors, $70 \%$ vs $42 \%$, respectively $(P<0.001)$. Seven patients were originally graded as low grade according the WHO2004 grading system. These seven patients were excluded in multivariate Cox-regression analysis.

\section{Validation of the Prognostic Markers for Prediction of Progression}

Tumor DNA was analyzed for methylation of GATA2, TBX2, TBX3, and ZIC4. Methylation analysis failed in $41 / 768(5 \%)$, ie GATA2 $n=0, T B X 2$ $n=20, T B X 3 n=14$, and ZIC4 $n=7$. The efficacy of each gene was determined by calculating the area under the receiver-operating curve. The area under the curve for GATA2 was 0.803, TBX2 0.644, TBX3 0.785 , and ZIC4 0.692. A methylation percentage

Table 2 Patient and tumor characteristics

\begin{tabular}{lccc}
\hline & $\begin{array}{c}\text { Progressors } \\
\mathrm{n}=77\end{array}$ & $\begin{array}{c}\text { Non-progressors } \\
\mathrm{n}=115\end{array}$ & \\
\hline $\begin{array}{l}\text { Age } \\
\text { Mean (range) }\end{array}$ & $68(47-85)$ & $63(23-86)$ & \\
& $\mathbf{n}(\%)$ & $\mathbf{n}(\%)$ & P-value \\
\hline $\begin{array}{l}\text { Sex } \\
\text { Male }\end{array}$ & $59(77)$ & $84(73)$ & 0.58 \\
Female & $18(23)$ & $31(27)$ & \\
Stage & & & - \\
$\quad$ Ta & $77(100)$ & $115(100)$ & \\
Grade & & & \\
$\quad$ Grade 1 & & & \\
Grade 2 & $18(23)$ & $49(56)$ & \\
Low grade & $54(70)$ & $2(2)$ & \\
\hline
\end{tabular}

cut-off was selected with the best combination of sensitivity and specificity for each individual marker (Figure 1). Cut-off values, sensitivity, and specificity are given in Table 3 . To determine the predictive value of the methylation markers for developing progression over time, log-rank analysis was performed based on a follow-up period of 10 years. 66/77 (86\%) of the progressors developed disease progression within 10 years of followup. Figure 2 shows the resulting survival graphs for grade and the four methylation markers. Patients with a G1 tumor or low methylation of TBX2, TBX3, GATA2, and ZIC4 had a significantly better progression-free survival, $P<0.001$ (Figure 2). In order to calculate the combined effect of the methylation markers for predicting progression over time, multivariate Cox-regression analysis with backward elimination was performed. This analysis based on TBX2, TBX3, GATA2, and ZIC4 in combination with grade showed the clear-independent prognostic ability of $T B X 3$ for the risk of progression within 10 years of follow-up time (Table 4, multivariate analysis step \#1). After removing GATA2 as predictor from the model TBX3 and TBX2 appeared the best predictors (multivariate analysis step \#2).

On the basis of the methylation status of TBX2 and $T B X 3$, a new molecular grading system was defined. Patients were assigned to the high molecular grade group in case both the markers were positive. The intermediate grade group included patients with one marker positive. The low molecular grade group consisted of patients with none of the markers positive. Disease progression occurred in $32 / 43(74 \%)$ of the patients in the high molecular grade group and in 20/49 (40\%) patients of the intermediate-molecular grade group. In comparison, only $9 / 73(12 \%)$ patients in the low molecular grade group showed disease progression. The majority of patients developed progression within 5 years of followup: $27 / 43(63 \%)$ of the patients from the high molecular grade group compared with 14/49 (29\%) of the intermediate grade group and $6 / 73(8 \%)$ of the low molecular grade group. Log-rank analysis showed significantly better progression-free survival in patients assigned to the low molecular grade group compared with patients at the intermediatemolecular grade group and the high molecular grade group. Furthermore, patients in the intermediate grade group showed better progression-free survival than patients in the high molecular grade group (low molecular grade vs intermediate-molecular grade $P=0.04$, low molecular grade group vs high molecular grade $P<0.001$, intermediate-molecular grade vs high molecular grade $P<0.001$; Figure 3 ).

\section{Reevaluation of the Pathological Review}

All cases were reviewed by an expert uropathologist. Seventeen were not available. This reevaluation was not completely unbiased because the 

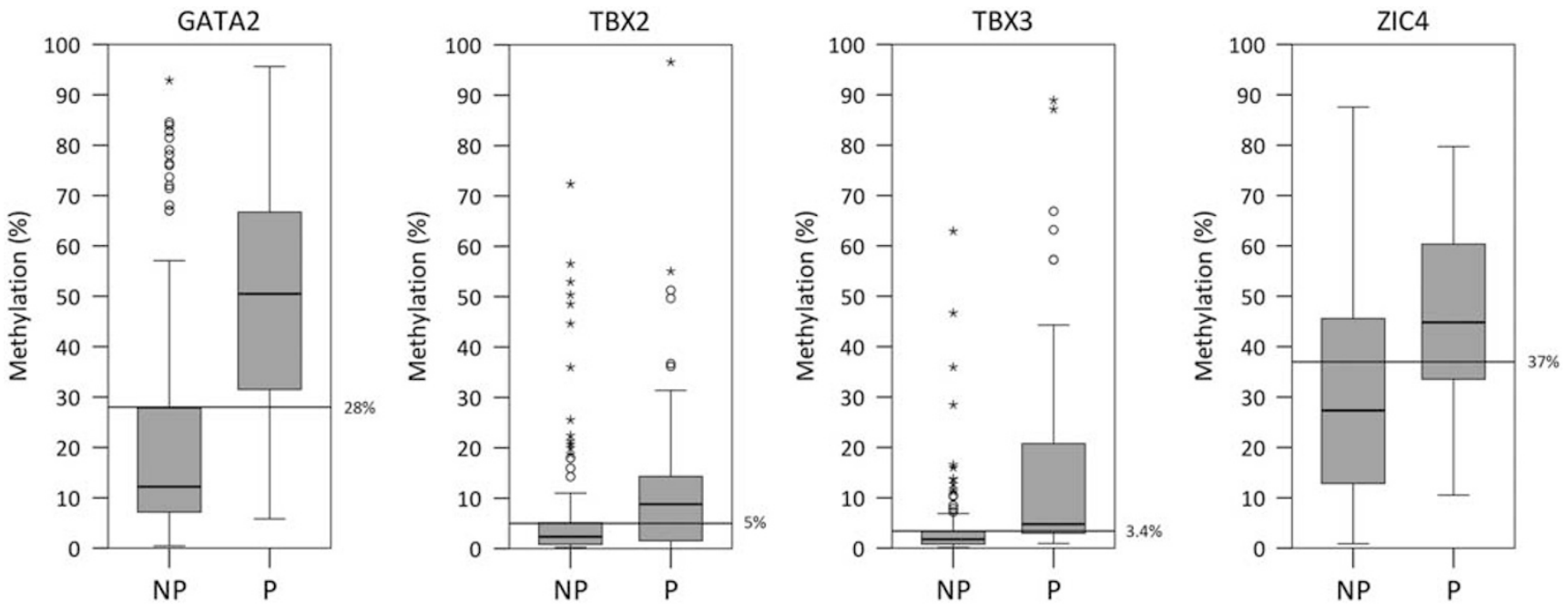

Figure 1 Boxplots indicating the range of gene methylation percentage (\%) regarding the progression status: progressors (P) and nonprogressors (NP). The horizontal line indicates the cut-off.

Table 3 Methylation marker performance for predicting disease progression

\begin{tabular}{|c|c|c|c|c|c|c|}
\hline Methylation marker & Cut-off & Sensitivity & $\mathrm{n}$ & Specificity & $\mathrm{n}$ & $A U C$ \\
\hline TBX3 & $3.4 \%$ & $71 \%$ & $51 / 72$ & $76 \%$ & $80 / 106$ & 0.785 \\
\hline$T B X 2$ & $5.0 \%$ & $63 \%$ & $46 / 73$ & $66 \%$ & 66/99 & 0.644 \\
\hline ZIC4 & $37 \%$ & $70 \%$ & $53 / 76$ & $64 \%$ & 70/109 & 0.692 \\
\hline GATA2 & $28 \%$ & $78 \%$ & $60 / 77$ & $75 \%$ & $86 / 115$ & 0.803 \\
\hline
\end{tabular}

pathologist was aware that the series comprised many tumors that had progressed. Reassessment of the histological slides revealed a dissimilarity of $12 \%(21 / 175)$ in staging and of $54 \%(95 / 175)$ in revised grading by the WHO1973 system. 80/175 $(45 \%)$ tumors were upgraded and $8 \%(15 / 175)$ of the tumors were downgraded. Furthermore, tumors of the progressors were higher graded and staged compared with the group of non-progressors, $P<0.001$ (Table 5). Revised grade by the WHO2004-system showed more high-grade tumors in the progressor group $(P<0.001)$.

Log-rank analysis based on the revised WHO1973 grade revealed better progression-free survival in G1 vs G2 and G2 vs G3 (Supplementary Figure 1A; G1 vs G2 $P=0.026$, G1 vs G3 $P<0.001$, and G2 vs G3 $P<0.001)$. WHO2004 grading also showed a worse progression-free survival for patients with a highgrade tumor compared with patients with a low grade or PUNLMP tumor (Supplementary Figure 1B; HG vs LG $<0.001$, LG vs PUNLMP $P=0.534$, and HG vs PUNLMP $P=0.035)$. Next, we performed multivariate Cox-regression analyses with backward elimination to compare revised grade with the molecular markers. These results are depicted in the Supplementary Tables 1 and 2. Compared with both grading systems, TBX3 and TBX2 remained the best predictors for progression.

\section{Discussion}

Although, most important predictors for disease progression in patients with non-muscle-invasive bladder cancer are stage, grade, and carcinoma in situ, ${ }^{18}$ patients with primary low-stage and lowgrade bladder cancer still have a $1-6 \%$ chance of disease progression according the European Association of Urology guidelines. ${ }^{3}$ In order to safely reduce the number of cystoscopies in patients with low grade non-muscle-invasive bladder cancer, risk prediction is necessary. Up to now, no sensitive markers are available to predict progression in this specific patient group. Recently, Kandimalla et al. ${ }^{16}$, proved the prognostic ability of the methylation markers TBX2, TBX3, GATA2, and ZIC4 using freshfrozen tumor tissue. ${ }^{16}$ In this more extensive validation, these markers again proved to be highly sensitive for prediction of progression in low-grade pTa disease. Patients with low methylation percentages of TBX2, TBX3, GATA2, and ZIC4 showed better progression-free survival in univariate logrank analysis. Multivariate Cox-regression analysis showed an independent increased risk for progression in patients with high methylation ratios of TBX2, TBX3, and ZIC4. New molecular grading, based on the methylation status of TBX2 and TBX3, into three risk groups resulted in accurate prediction 
a

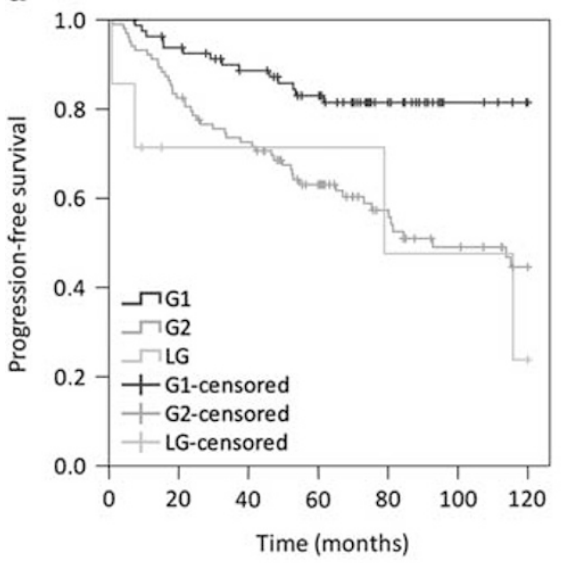

b

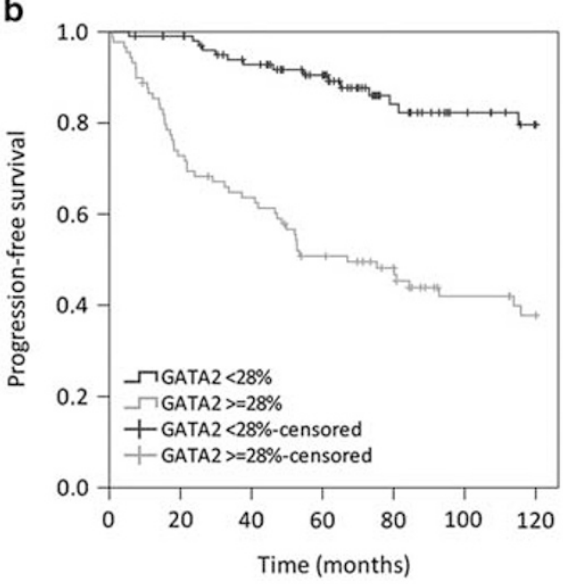

C

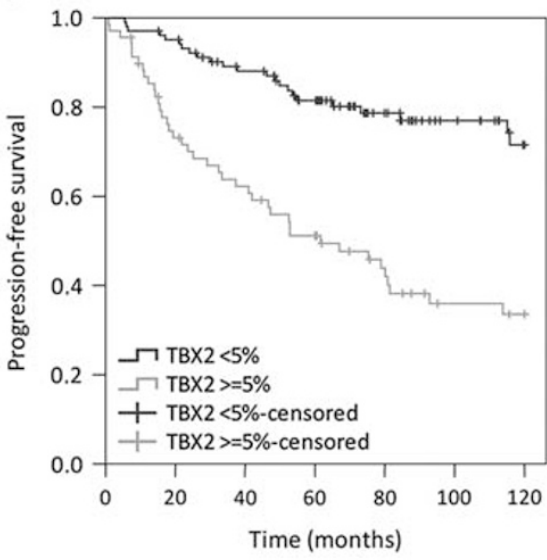

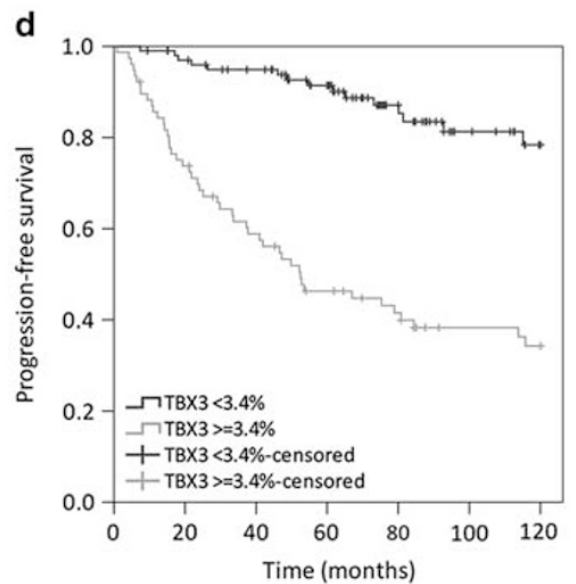

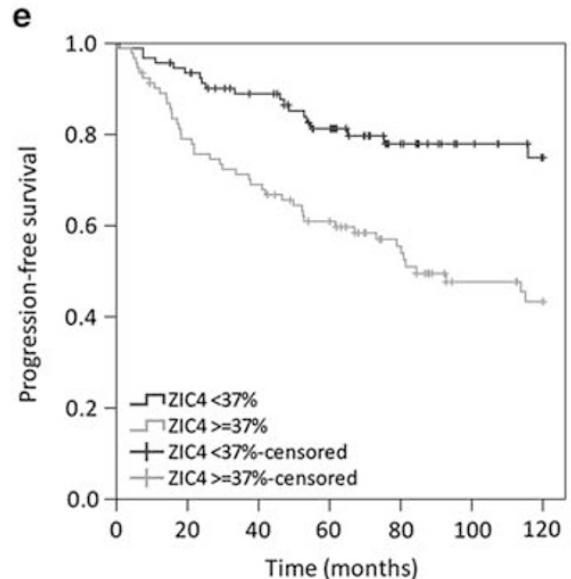

Figure 2 Kaplan-Meier curves showing the time to progression of patients with primary TaG1/G2 BC, according to the grade (a) given at time of diagnosis and according to the methylation status of GATA2 (b), TBX2 (c), TBX3 (d) and ZIC4 (e) in the primary tumor. (a): G1 vs G2 $P<0.001$, G1 vs LG $P<0.001$, and G2 vs LG $P=0.355$. (b-e): patients with a low methylation status showed better progression-free survival compared with the patients with high methylation status $(P<0.001)$.

Table 4 Univariate and multivariate progression-free survival analysis

\begin{tabular}{|c|c|c|c|c|c|c|c|c|c|}
\hline \multirow[b]{2}{*}{ Variable } & \multicolumn{3}{|c|}{ Univariate analysis } & \multicolumn{3}{|c|}{ Multivariate step \#1 } & \multicolumn{3}{|c|}{ Multivariate step \#2 } \\
\hline & No of pts & Events & $\mathrm{P}$-value & $H R$ & $95 \% C I$ & $\mathrm{P}$-value & $H R$ & $95 \% C I$ & $\mathrm{P}$-value \\
\hline \multicolumn{10}{|l|}{ Grade } \\
\hline G1 & 82 & 14 & $<0.001$ & 1.824 & $0.957-3.474$ & 0.068 & 1.882 & $0.988-3.586$ & 0.054 \\
\hline G2 & 103 & 48 & & & & & & & \\
\hline \multicolumn{10}{|l|}{$T B X 2$} \\
\hline$<5.0 \%$ methylated & 103 & 23 & $<0.001$ & 1.656 & $0.922-2.975$ & 0.091 & 1.873 & $1.069-3.283$ & 0.028 \\
\hline$\geq 5.0 \%$ methylated & 69 & 41 & & & & & & & \\
\hline \multicolumn{10}{|l|}{ TBX3 } \\
\hline$<3.4 \%$ methylated & 101 & 15 & $<0.001$ & 3.522 & $1.788-6.936$ & $<0.001$ & 4.087 & $2.149-7.772$ & $<0.001$ \\
\hline$\geq 3.4 \%$ methylated & 77 & 47 & & & & & & & \\
\hline \multicolumn{10}{|l|}{ ZIC4 } \\
\hline$<37 \%$ methylated & 93 & 19 & $<0.001$ & 1.786 & $0.985-3.237$ & 0.056 & 1.873 & $1.038-3.379$ & 0.037 \\
\hline$\geq 37 \%$ methylated & 92 & 46 & & & & & & & \\
\hline \multicolumn{10}{|l|}{ GATA2 } \\
\hline$<28 \%$ methylated & 103 & 15 & $<0.001$ & 1.605 & $0.802-3.213$ & 0.181 & - & - & - \\
\hline$\geq 28 \%$ methylated & 89 & 51 & & & & & & & \\
\hline
\end{tabular}




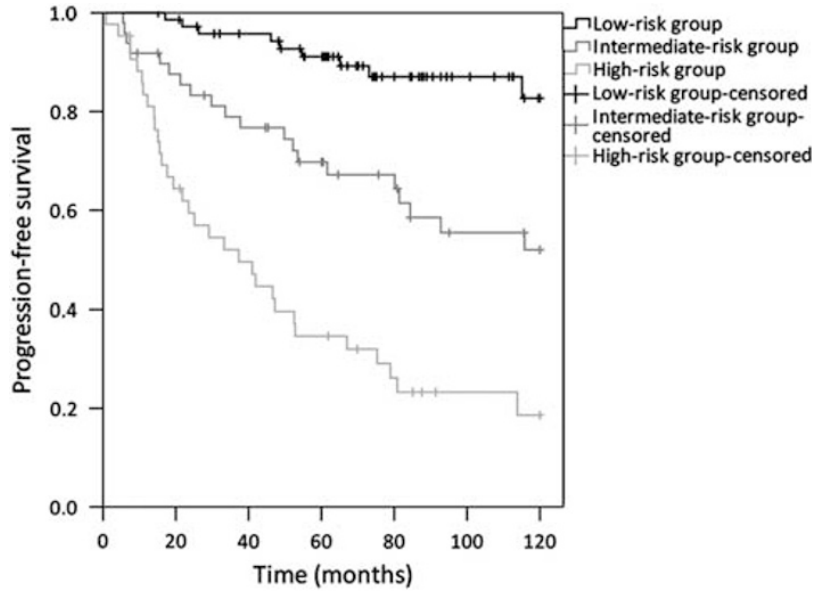

Figure 3 Patients stratified according to methylation status of $T B X 2$ and TBX3 into three molecular grade groups: low molecular grade (lmG), both markers negative; intermediate-molecular grade (imG), one marker positive; and high molecular grade (hmG), both markers positive. A follow-up time of maximum 10 years was selected.

Table 5 Tumor characteristics after reassessment of histological slides

\begin{tabular}{|c|c|c|c|}
\hline & Progressors & Non-progressors & \\
\hline Reassessment & $\begin{array}{l}\mathrm{n}=77 \\
\mathrm{n}(\%)\end{array}$ & $\begin{array}{c}\mathrm{n}=98 \\
\mathrm{n}(\%)\end{array}$ & $\mathrm{P}$-value \\
\hline \multicolumn{4}{|l|}{ Stage } \\
\hline Papilloma & $2(3)$ & $1(1)$ & \multirow{5}{*}{$<0.001$} \\
\hline Та & $56(73)$ & 95 (97) & \\
\hline $\mathrm{T} 1$ & $17(22)$ & $2(2)$ & \\
\hline $\mathrm{T} 2$ & $1(1)$ & - & \\
\hline Tis & $1(1)$ & - & \\
\hline \multicolumn{4}{|c|}{ Revised grade by WHO $1973^{\mathrm{a}}$} \\
\hline Papilloma & $2(3)$ & $1(1)$ & \multirow[t]{4}{*}{$<0.001$} \\
\hline G1 & $3(4)$ & $21(22)$ & \\
\hline G2 & $45(59)$ & 66 (67) & \\
\hline G3 & $26(34)$ & $10(10)$ & \\
\hline \multicolumn{4}{|c|}{ Revised grade by WHO 2004 } \\
\hline Papilloma & $2(3)$ & $1(1)$ & \multirow[t]{4}{*}{$<0.001$} \\
\hline PUNLMP & $2(3)$ & $8(8)$ & \\
\hline Low grade & $20(26)$ & $57(58)$ & \\
\hline High grade & $52(68)$ & $32(33)$ & \\
\hline
\end{tabular}

${ }^{\mathrm{a}}$ Without Tis $n=1$.

of progression risk, with better progression-free survival in patients in the low risk group and intermediate risk group. In addition, this validation was carried out on DNA isolated from formalin-fixed paraffin-embedded tissue.

The four investigated genes, TBX2, TBX3, GATA2, and ZIC4, encode transcription factors that are important in the regulation of developmental processes and gene expression is more frequent in nonmuscle-invasive bladder cancer compared with muscle-invasive disease. ${ }^{19,20}$ This could be linked to our results in which aggressive tumors that progressed to muscle-invasive bladder cancer showed more methylation than the non-progressive tumors. Furthermore, the most significant markers, $T B X 2$ and $T B X 3$, downregulate the p53-pathway by inhibiting the expression of the $A R F$ and CIP1 gene. ${ }^{21}$ Mutation of the p53 gene is a common phenomenon in muscle-invasive bladder cancer. Methylation of TBX2 or TBX3 could be a different way to inhibit the p53-pathway. Therefore, we propose that methylation of TBX2 and TBX3 characterizes the more aggressive non-muscleinvasive bladder cancer, which are more similar to the muscle-invasive bladder cancer.

Multiple studies, previously reported the prognostic ability of methylation markers based on DNA isolated from formalin-fixed paraffin-embedded tisue. ${ }^{22}$ Friedrich et $a l .{ }^{23}$, found that methylation of TIMP3 was associated with a better progression-free survival in patients with pT1 and pTa tumors. Yan et al. ${ }^{24}$, showed that methylation of RUNX3 in combination with G3 increased the chance of disease progression. According to Yates et al. ${ }^{15}$, methylation of E-cadherin, TNFRSF25, EDNRB, $R A S S F 1 A$, and $A P C$ were significantly associated with progressive disease. Yet, these studies all used a mixture of pT1 and pTa tumors. Our study specifically focused on patients with low-grade pTa disease. These patients have the lowest chance of developing progression and therefore, adjustment of the surveillance protocol will be most beneficial in this particular patient group.

The progression scores as recommended in the European Association of Urology guidelines are currently calculated according to the European Organisation of Research and Treatment of Cancer risk scores developed by Sylvester et al. ${ }^{3}$ The pathological review has an important role in this prediction model. However, this pathological assessment highly depends on the experience of the pathologist and suffers from high-interobserver and -intraobserver variation. ${ }^{8,25}$ Upgrading and upstaging could increase the 5-year progression risk up to $45 \%$-according to the European Organisation of Research and Treatment of Cancer risk calculator. This emphasizes the importance of accurate pathological assessment. This study again proved the high-interobserver variation with significant more high-grade tumors among the progressors after pathological reassessment. Yet, it should be emphasized that the pathological review could be biased. Although, the slides were blinded, the pathologist was aware of the fact that there was a high percentage of aggressive tumors in this study. Therefore, we decided to use the original pathological grade as inclusion criteria, as this is the diagnosis on which further treatment was based. Patient outcome is a result of this original review and the treatment choices made.

The use of molecular markers could facilitate the pathologist's estimation of tumor aggressiveness. 
A previous study by van Rhijn et al. ${ }^{26}$ investigated the additional value of molecular markers to the pathological assessment. Molecular grading based on the combination of FGFR3 mutation status and MIB1 expression resulted in better risk prediction compared with conventional pathological grade. The addition of the methylation markers to FGFR3 and MIB1 might further increase the predictive accuracy. The methylation analysis is an easy test and could be performed in a standard laboratory. Furthermore, the Snapshot method proved to be highly reproducible. ${ }^{27}$

A limitation of this study is the retrospective design. Because of the low incidence of progression in low-grade pTa disease a prospective study design is difficult to establish. Therefore, the distribution of patients is not representative for the clinical practice, since the actual percentage of patients with low-grade non-muscle-invasive bladder cancer and disease progression will be much lower in the clinical setting. However, the predictive risk ability of the methylation markers still holds after a second validation and therefore, we believe that these results could be translated to the clinic.

In conclusion, this study represents an independent validation of pTa-prognostic markers in a large set of patients. These markers proved again to be accurate risk predictors for progression in patients with primary low-grade pTa-bladder cancer. New molecular grading based on the methylation status of $T B X 2$ and $T B X 3$, resulted in sensitive risk prediction for progression. Determining DNA methylation is a highly reproducible method as we showed previously. ${ }^{27}$ Hence these markers present a valuable tool for assessing risk of progression in patients presenting with TaG1/G2 tumors.

\section{Acknowledgments}

This study was supported by European Community Seventh Framework program FP7/2007-2012, Grant agreement no. 201663, Erasmus MC (MRace 2007), and Dutch Cancer Society Grant no. EMCR 2007-3863.

\section{Disclosure/conflict of interest}

The authors declare no conflict of interest.

\section{References}

1 Parkin DM, Bray F, Ferlay J, et al. Global cancer statistics, 2002. CA Cancer J Clin 2005;55:74-108.

2 van Rhijn BW, Burger M, Lotan Y, et al. Recurrence and progression of disease in non-muscle-invasive bladder cancer: from epidemiology to treatment strategy. Eur Urol 2009;56:430-442.

3 Sylvester RJ, van der Meijden AP, Oosterlinck W, et al. Predicting recurrence and progression in individual patients with stage Ta T1 bladder cancer using EORTC risk tables: a combined analysis of 2596 patients from seven EORTC trials. Eur Urol 2006;49:475-7.

4 van Rhijn BW, Zuiverloon TC, Vis AN, et al. Molecular grade (FGFR3/MIB-1) and EORTC risk scores are predictive in primary non-muscle-invasive bladder cancer. Eur Urol 2010;58:433-441.

5 van der Aa MN, Steyerberg EW, Sen EF, et al. Patients' perceived burden of cystoscopic and urinary surveillance of bladder cancer: a randomized comparison. BJU Int 2008;101:1106-1110.

6 Botteman MF, Pashos CL, Hauser RS, et al. Quality of life aspects of bladder cancer: a review of the literature. Qual Life Res 2003;12:675-688.

7 Van Der Meijden A, Sylvester R, Collette L, et al. The role and impact of pathology review on stage and grade assessment of stages Ta and T1 bladder tumors: a combined analysis of 5 European Organization for Research and Treatment of Cancer Trials. J Urol 2000; 164:1533-1537.

8 van Rhijn BW, van Leenders GJ, Ooms BC, et al. The pathologist's mean grade is constant and individualizes the prognostic value of bladder cancer grading. Eur Urol 2010;57:1052-1057.

9 Bol MG, Baak JP, Buhr-Wildhagen S, et al. Reproducibility and prognostic variability of grade and lamina propria invasion in stages $\mathrm{Ta}$, T1 urothelial carcinoma of the bladder. J Urol 2003;169:1291-1294.

10 Kurth KH, Sylvester RJ. Prognostic factors in nonmuscle- invasive bladder tumors. i. clinical prognostic factors: a review of the experience of the EORTC genito-urinary group ii. biologic prognostic markers. Eur Urol 2007;6:789-799.

11 Adshead JM, Kessling AM, Ogden CW. Genetic initiation, progression and prognostic markers in transitional cell carcinoma of the bladder: a summary of the structural and transcriptional changes, and the role of developmental genes. Br J Urol 1998;82:503-512.

12 Knowles MA. Molecular subtypes of bladder cancer: Jekyll and Hyde or chalk and cheese? Carcinogenesis 2006;27:361-373.

13 Catto JW, Azzouzi AR, Rehman I, et al. Promoter hypermethylation is associated with tumor location, stage, and subsequent progression in transitional cell carcinoma. J Clin Oncol 2005;23:2903-2910.

14 Marsit CJ, Houseman EA, Schned AR, et al. Promoter hypermethylation is associated with current smoking, age, gender and survival in bladder cancer. Carcinogenesis 2007;28:1745-1751.

15 Yates DR, Rehman I, Abbod MF, et al. Promoter hypermethylation identifies progression risk in bladder cancer. Clin Cancer Res 2007;13:2046-2053.

16 Kandimalla R, van Tilborg AA, Kompier LC, et al. Genome-wide analysis of CpG island methylation in bladder cancer identified TBX2, TBX3, GATA2, and ZIC4 as pTa-specific prognostic markers. Eur Urol 2012;61:1245-1256.

17 Casparie M, Tiebosch AT, Burger G, et al. Pathology databanking and biobanking in The Netherlands, a central role for PALGA, the nationwide histopathology and cytopathology data network and archive. Cell Oncol 2007;29:19-24.

18 van Rhijn BW. Combining molecular and pathologic data to prognosticate non-muscle-invasive bladder cancer. Urol Oncol 2012;30:518-523.

19 Smith J. T-box genes: what they do and how they do it. Trends Genet 1999;15:154-158. 
20 Sanchez-Carbayo M, Socci ND, Lozano J, et al. Defining molecular profiles of poor outcome in patients with invasive bladder cancer using oligonucleotide microarrays. J Clin Oncol 2006;24:778-789.

21 Lu J, Li XP, Dong Q, et al. TBX2 and TBX3: the special value for anticancer drug targets. Biochim Biophys Acta 2010;1806:268-274.

22 Kandimalla R, van Tilborg AA, Zwarthoff EC. DNA methylation-based biomarkers in bladder cancer. Nat Rev Urol 2013;10:327-335.

23 Friedrich MG, Chandrasoma S, Siegmund KD, et al. Prognostic relevance of methylation markers in patients with non-muscle invasive bladder carcinoma. Eur J Cancer 2005;41:2769-2778.

24 Yan C, Kim YW, Ha YS, et al. RUNX3 methylation as a predictor for disease progression in patients with non-muscle-invasive bladder cancer. J Surg Oncol 2012;105:425-430.

25 May M, Brookman-Amissah S, Roigas J, et al. Prognostic accuracy of individual uropathologists in noninvasive urinary bladder carcinoma: a multicentre study comparing the 1973 and 2004 World Health Organisation classifications. Eur Urol 2010;57:850-858.

26 van Rhijn BW, Vis AN, van der Kwast $\mathrm{TH}$, et al. Molecular grading of urothelial cell carcinoma with fibroblast growth factor receptor 3 and MIB-1 is superior to pathologic grade for the prediction of clinical outcome. J Clin Oncol 2003;21:1912-1921.

27 Kandimalla R, Masius R, Beukers W, et al. A 3-plex methylation assay combined with the FGFR3 mutation assay sensitively detects recurrent bladder cancer in voided urine. Clin Cancer Res 2013;19:4760-4769.

Supplementary Information accompanies the paper on Modern Pathology website (http://www.nature.com/ modpathol) 